\title{
EFFECT OF HYSTERESIS IN AXIAL COMPRESSORS OF GAS-TURBINE ENGINES
}

\author{
${\text { Mykola Kulyk }{ }^{1} \text {, Ivan Lastivka }{ }^{2} \text {, Yuri Tereshchenko }}^{3}$ \\ National Aviation University, 1 Kosmonavta Komarova Ave., 03680 Kiev, Ukraine \\ E-mail:2eduicao@nau.edu.ua (corresponding author)
}

Received 19 March 2012; accepted 22 October 2012

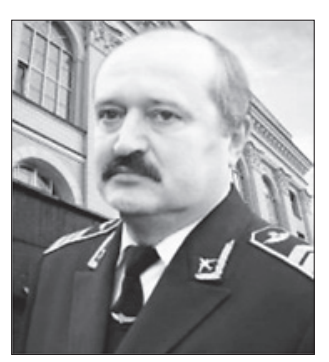

Mykola KULYK, Prof. Dr Sci

Education: Kiev Institute of Civil Aviation Engineers, 1976. In 1993 - Doctor of Science (Engineering). In 1994 - Professor.

Affiliations and functions: 1997-head of the Department of Aircraft Engines, 2008rector of the National Aviation University.

Honours, awards: Honoured Figure of Science and Engineering of Ukraine, winner of the State Prize of Ukraine in the area of science and technology.

Research interests: automatic systems of aircraft engine technical conditions.

Publications: over 200 scientific papers.

Present position: rector of the National Aviation University, Ukraine.

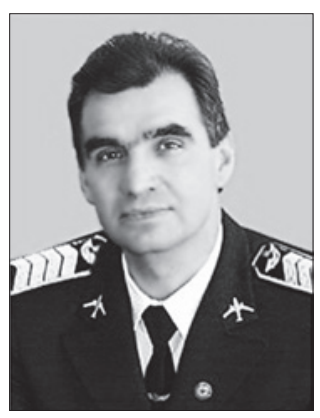

Ivan LASTIVKA, $P h D$

Education: Taras Shevchenko National University of Kiev, Faculty of Mechanics and Mathematics, 1982.

Affiliations and functions: since 2006 - head of the Higher Mathematics Department. Research interests: models and methods to improve the aerodynamic and aeroelastic characteristics of the compressors of gas-turbine engines.

Publications: 78 scientific papers.

Present position: head of the Higher Mathematics Department, National Aviation University.

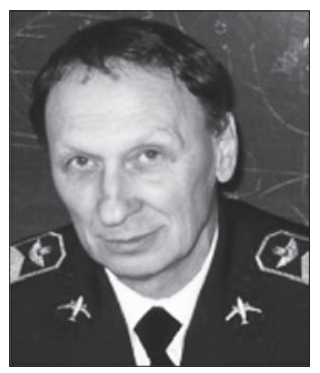

Yuri TERESHCHENKO, Prof. Dr Sci

Education: Kiev Institute of Air Force, 1962. In 1981 - Doctor of Science (Engineering). Affiliations and functions: since 2000 - professor in the Department of Aircraft Engines. Honours, awards: Honoured Figure of Science and Engineering of Ukraine, winner of the State Prize of Ukraine in the area of science and technology.

Research interests: enhancement of the performance, efficiency and technical resources of aircraft power plants.

Publications: over 200 scientific papers.

Present position: professor in the Department of Aircraft Engines, the National Aviation University.

Abstract. The phenomenon of separated flow hysteresis in the process of the streamlining the axial compressor of gas-turbine engines is considered. Generalised results of research on the occurrence of hysteresis in the aerodynamic performance of compressor grids and its influence on the performance of the bladed disks of compressors that operate in real conditions of periodic circular non-uniformity are demonstrated.

Keywords: gas-turbine engines, axial compressor, hysteresis. 


\section{Introduction}

The operation of gas-turbine engine compressor aerofoil grids at supercritical angles of attack (angles of flow acceleration) is connected with the appearance of intensive areas of flow separation on the blade surfaces. As a result, the values of the flow deflection angle and lift force coefficient sharply decrease and total pressure losses and grid aerofoil resistance significantly increase. Research of the occurrence of this process and development of flow separation modes in grids demands solving tasks connected with predicting the aforementioned occurrence of flow separation modes in compressor grids, defining ways to extend the range of the non-separated streamline of the bladed disks of compressors, and increasing their gas-dynamic stability margins (Chang 1979; Tereshchenko et al. 2005, 2010; Lastivka 2011).

The structure and characteristics of separated flows significantly depend on their kinematic performances velocity, angles of attack, and flow parameters such as pressure, density and temperature - and it depends on the direction of their change within the interval of certain values. This means that at the same ensemble of parameters different aerodynamic performances can emerge.

One of the reasons for this phenomenon is aerodynamic hysteresis brought about by the flow structure reforming when there is a change in at least one parameter (such as velocity of non-disturbed flow, angle of attack, or Reynolds's number) and under conditions of unsteady flow-frequency and amplitude of oscillations.

\section{Hysteresis at unsteady flow about aerofoils}

Since the aerodynamic performance of compressor grids is mostly defined by the processes that occur at the boundary layers on blades, there is great interest in considering the influence of periodic flow non-uniformity in front of grids on the compressor grids streamlining and flow at the boundary layer on blades (Schlichting 1969; Liblein, Randebuseh 1956).

Taking into consideration the flow in bladed disks of multistage compressors operating under conditions of periodic angular flow non-uniformity caused by disturbances created by the aerodynamic wake behind the trailing edges of blades of previous stages, it is possible to specify that the calculation pattern that corresponds to the image of non-steady flow about the compressor grid can be obtained if the values of velocities and pressures are presented in the form of the sum of steady and pulsating components:

$$
\begin{aligned}
& u(x, y, \tau)=\bar{u}(x, y)+u_{1}(x, y, \tau) \\
& v(x, y, \tau)=\bar{v}(x, y)+v_{1}(x, y, \tau) \\
& p(x, \tau)=\bar{p}(x)+p_{1}(x, \tau),
\end{aligned}
$$

where $\bar{u}(x, y), \bar{v}(x, y), \bar{p}(x)$ are averaged by the period values of the components of velocities $u$ and $v$ and pressure $p$; and $u_{1}(x, y, \tau), v_{1}(x, y, \tau)$, and $p_{1}(x, \tau)$ are pulsating components of velocities and pressure. The axes of coordinates $\mathrm{x}$ and $\mathrm{y}$ in this case are directed correspondingly along the aerofoil chord and perpendicularly to it. The method provides linearization of the equation, which determines the velocity of periodic flow in the main stream. Analysis of the theoretical solution of the problem regarding the definition of pulsating velocity field (Stokes' problems regarding infinite plate streamlining by oscillating flow) in the form of Stokes' waves demonstrates that different layers oscillate with a phase shift relating to the outer flow and relative to each other. It stipulates the inertness of transient processes in the boundary layer and the possibility of the occurrence of hysteresis at the process of plate streamlining by the periodically non-uniform flow. The core of the solution is in the fact that they suggest deformation of only the external flow to the border of the boundary layer at high-frequency disturbances that overlap the main flow. The flow in the core of the boundary layer does not change significantly in comparison with the surface streamlining by steady flows. At low-frequency disturbances in the flow that correspond to the values of Strouhal number $\mathrm{S}_{r}<1$, flow character change occurs in the boundary layer, which means a change in its structure.

The influence of external flow pulsation on the velocity and temperature profiles of steady mean motion is studied by I. Kestin, P. Maeders and H. Wang (Kestin et al. 1961). In this work, the behaviour of the boundary layer is considered under the condition that the velocity on the boundary is given as follows:

$$
W=W_{0}\left[1-\lambda \cos \omega\left(\frac{x}{W_{0}}-\lambda\right)\right],
$$

where $W_{0}$ is the speed of undisturbed flow, $x$ is a specific dimension of a streamlined body, $\omega$ is an angular frequency, and $C_{o p}^{m}$ is a speed parameter.

Solving equations of unsteady boundary layer by applying such a velocity profile allows separating the steady component of the dynamic boundary layer and harmonics of the second order. Thus, harmonisation of the flow non-uniformity (external disturbances that overlap steady flow) allows simplifying the solution to theoretical problems and preliminarily predicting when it is possible to apply the principle of flow parameter averaging for research of the flow in the layers near the walls.

From the abovementioned analysis of the theoretical research results of unsteady boundary layers, essentially important conclusions are made about the fact that the most significant influence on the flow in the boundary 
layer and, especially, in the boundary layer areas near the walls is caused by low-frequency oscillations that have an impact on the main stream and high-frequency disturbances but do not affect the boundary layer flow. Common to all theoretical research is the application of a set of empirical ratios for solving the equations that describe the processes in unsteady boundary layers. The trustworthiness and correctness of the ratios are determined by the quality of the theoretical solutions obtained.

In this work, the results of research of drag of circular cylinders during acceleration are discussed. Acceleration or deceleration in the flow that moves around the cylinder causes significant change to the structure of the boundary layer and, correspondingly, change to the position of the point of boundary layer separation: during acceleration of the flow around the cylinder $(\partial W / \partial \tau)>0$, the point of separation shifts backwards, causing a decrease in aerodynamic trail width and as a result profile drag caused by viscous forces in the flow (the coefficient of profile drag $C_{x}$ decreases); during deceleration in the flow $(\partial W / \partial \tau)<0$, the point of boundary layer separation shifts forward, widening the aerodynamic trail behind the cylinder and increasing profile drag. These conclusions characterise changes in the instantaneous coefficient of the profile drag of the cylinder, which oscillates in the flow.

In the influence of relative acceleration of the flow on the aerofoil, aerodynamic performance is investigated, and it is shown that acceleration enhances non-separated flow about the surfaces and deceleration initiates flow separation.

It is quite evident that there is an analogy between this phenomenon and the streamlining of the compressor grids by periodically non-uniform flow. When the angle of attack is increased (deceleration of the flow), the separation point moves to the leading edge of a blade, and during decrease in angle of attack (flow acceleration), it moves to the trailing edge, and correspondingly the coefficient of grid profile drag $C_{x}$ periodically changes.

In aerofoil grids that are streamlined by periodic non-uniform flow under some certain conditions, the transition of grids to supercritical modes of operation with further recovery or non-recovery of the initial mode is a possibility. Transition of grids to supercritical modes and their return to subcritical modes are usually accompanied by hysteresis. In the transition from separated mode to non-separated mode of streamlining, the compressor grid does not return along the normal characteristic (Fig. 1, graph $a$ ) but along a separated one (Fig. 1 , graph $\delta$ ).

Research of the separation modes of the operation of axial compressor stages demonstrated that for each frequency of rotor rotation there are two different characteristics, two pressure lines: one of them corresponds to non-separated flow about bladed disks (Fig. 2, curve a) and another one corresponds to flow separation (Fig. 2 , curve $\delta$ ).

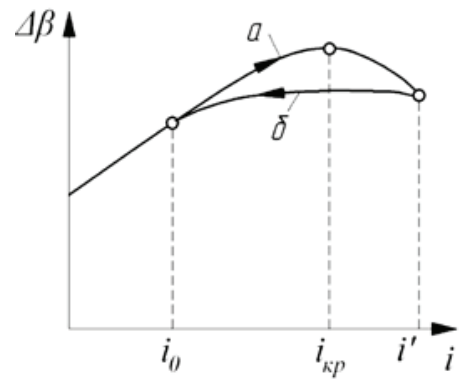

Fig. 1. Hysteresis of diffuser grid performance

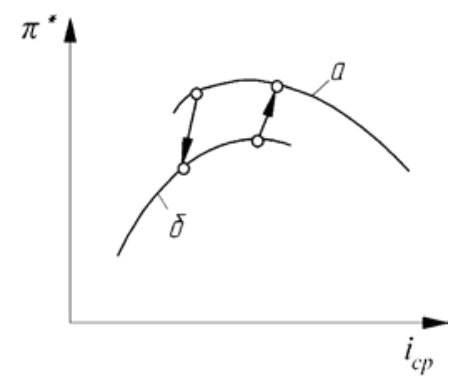

Fig. 2. Hysteresis of axial compressor stage performance

Axial compressor stage performance is presented by the functional dependence of pressure ratio $\pi^{*}$ on the reduced frequency of rotor rotation $\overline{n_{\text {red }}}$ and averaged by the value of the blade height of the angle of attack $i_{a v}$ of the bladed disk grid. The non-separated pressure line (a) in figure 2 characterises flow about bladed disks at subcritical modes without flow separation processes. Separated characteristics (b) correspond to a steady mode of flow about bladed disks. During a surge, the compressor periodically passes from non-separated performance to separated and back. Passage from separated to non-separated flow performance occurs at lower angles of attack than the critical angles of attack that correspond to the beginning of flow separation. This phenomenon, known as hysteresis in compressor performance, has at its base some certain peculiarities in the process of the streamlining of compressor grids at subcritical and supercritical modes.

There is an interesting issue regarding the appearance of hysteresis in the performance of compressor grids that have different geometrical parameters.

Analysing the non-stationary compressor process grids overflow is recommended after a detailed study of hysteresis in the performance of the compressor grids at supercritical mode of operation under the quasi-stationary model. This is true when both kinematic flow parameters and aerofoil characteristics take place. Generalisation of the research results provides the opportunity to analyse the influence of the change in flow about blades on the peculiarities of normal and separated aerodynamic performances of grids and to arrive at the analysis of the influence of periodic non-uniformity of 
flow on the separation characteristics of grids. In general, the matter at hand is not about researching the influence of the change in the Strouhal number on the instantaneous values of lift and profile drag coefficients or flow deflection angle and coefficient of total pressure loss that characterise the efficiency of compressor grids but is about researching the influence of the Strouhal number on the display of hysteresis in the performance of compressor grids. A key to explaining the effects of hysteresis is comparison of the periodic separation frequency of vortices from the streamlined surface and frequency of flow oscillation (frequencies of the forced oscillations of blades in the flow). Research has established that the frequency of the separation of vortices (the frequency of the velocity pulsations in the aerodynamic trail behind the cylinder) corresponds to the values of Strouhal number $S_{\eta}=\left(\omega_{1} d / W\right)>0.2-0.4$ at values of the Reynolds number that are contained within the range of $0.3 \cdot 10^{6}<\operatorname{Re}<0.8 \cdot 10^{6}$, where $\omega_{1}$ is frequency of the separation of vortices, $d$ is diameter of a cylinder, and $W$ is speed of incoming airflow (Schlichting 1969; Kestin et al. 1961).

In figure 3 the values of Strouhal aerodynamic numbers are given. They characterise the periodicity of the separation of vortices at supercritical flow over cylinders. These numbers were obtained by different authors.

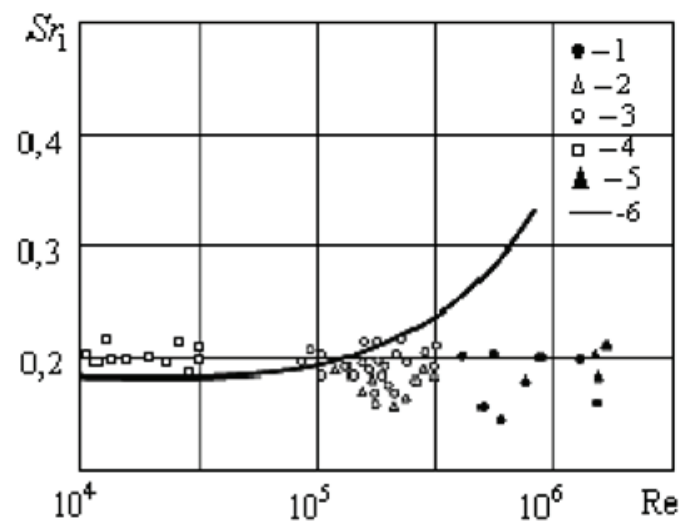

Fig. 3. Dependence of Strouhal aerodynamic number on Reynolds' number for cylinder in flow

Analysis of dependences $S_{\eta}=f(\mathrm{Re})$ shows that, at forced oscillations of a circular cylinder in a flow that streamlines the cylinder with the speed of $\mathrm{W}$, if the frequency of the oscillations of the cylinder differs significantly from the frequency of the separation of vortices, that is if Strouhal aerodynamic number $S_{r}=\omega d / W$ is greater than kinematic Strouhal number $S_{r}=\omega d / W$, then vortices with a frequency of $\omega_{1}$ when $S_{\eta}=0.2$ continue separating from the side surface of the oscillating cylinder. In the analysis of the functional dependence of the Strouhal aerodynamic number on the kinematic Strouhal number, a set of specific modes of oscillating cylinder streamlining is emphasised:
- The first mode is characterised by $0 \leq S_{r} \leq 0.04$. At this mode, the frequency of periodic separation of vortices corresponds to Strouhal aerodynamic number $S_{r}=0.2$ (this mode of separations of vortices takes place during oscillating cylinder streamlining and motionless cylinder streamlining);

- The second mode corresponds with the values of kinematic Strouhal number $0.04 \leq S_{r} \leq 0.2$. Within this range, periodic oscillations of the cylinder begin to influence the frequency of vortices decelerating, which is affected by oscillating cylinder frequency;

- The third mode corresponds to the values of Strouhal number $0.2 \leq S_{r} \leq 0.28$ and is characterised by intensive influence on the frequency of vortices. At Strouhal number $S_{r}>0.28$, there is no research or recommendations regarding the behaviour of the flow on the cylinder surface and in the aerodynamic trail behind it. This domain of change in Strouhal numbers corresponds to the streamlining of the bladed disks of axial compressors and requires thorough research.

\section{Research into the effect of hysteresis on the aerodynamic performance of compressor grids}

Experimental research into the effect of hysteresis on the aerodynamic performance of compressor grids was carried out in an open-circuit wind tunnel.

The aim of the experiment was to determine quantitative dependences that characterise the hysteresis of the aerodynamic performance of compressor grids (in a quasi-stationary statement of research problems). Performance of grids was determined at airflow speeds that correspond to Mach numbers $\mathrm{M}=0.4,0.5,0.6$ and 0.7 and Strouhal numbers $S_{r}<0.01$.

The effect of hysteresis was determined by successive spinning of the grids up to supercritical modes and recovering non-separated flow. The change in grid flow mode (by angle of attack) was carried out by means of stepped turn of the whole grid shroud placed in the working area of the wind tunnel. Measurements were fulfilled at fixed values of the angle of attack that differed from each other by $\Delta_{i}=1^{0}-2^{0}$. Based on the results of experimental research, dimensionless pressure profiles are built. The value of dimensionless pressure coefficient $\bar{p}$ was calculated for every drain point by the following formula:

$$
\bar{p}=\left(p-p_{m}\right) /\left(\rho W_{m}^{2} / 2\right),
$$

where $p_{m}$ is pressure in undisturbed flow in front of the grid, $p$ is pressure on the surface, $W_{m}$ is speed of flow in front of the grid, and $\rho$ is density. 
Figure 4 shows typical pressure profiles of dimensionless pressure coefficient on the aerofoils in grids with solidity $b / t=0.5 ; 1.0 ; 2.0$ at $M=0.6$ at non-separated and flow separation modes of flow. The occurrence of flow separation mode (at increase in angle of attack) and restoration of non-separated flow (at decrease in angle of attack) was controlled by pressure distribution on the surfaces of drained blades.

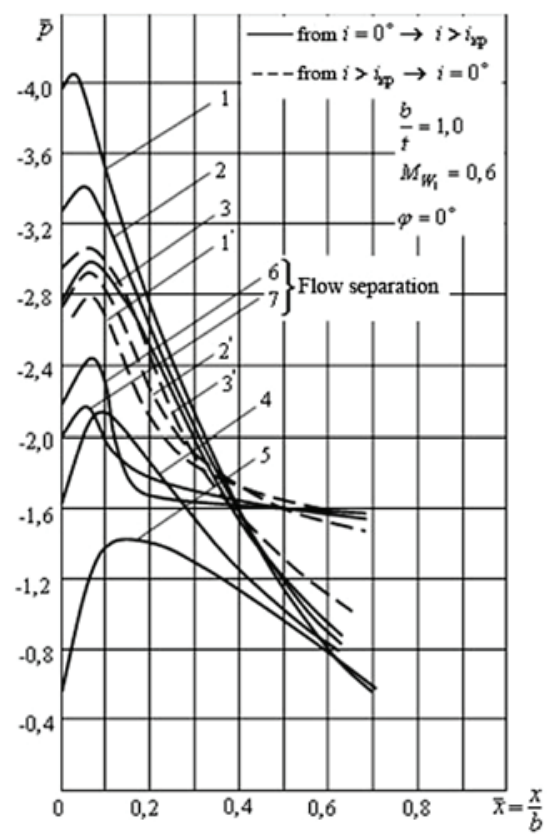

\begin{tabular}{|c|c|c|c|c|c|c|c|c|c|c|}
\hline $\begin{array}{c}\text { № } \\
\text { П/П }\end{array}$ & 1 & 2 & 3 & 4 & 5 & 6 & 7 & $1^{\prime}$ & 2 & 3 \\
\hline$i$ & $10 \neg$ & $8 \neg$ & $7 \neg$ & $5 \neg$ & $3 \neg$ & $14 \neg$ & $18 \neg$ & $10 \neg$ & $8 \neg$ & $7 \neg$ \\
\hline
\end{tabular}

Fig. 4. Pressure profiles of dimensionless pressure coefficient on the aerofoil in subsonic compressor grid

Specific modes that were used for the analysis of the phenomena of hysteresis are shown in figure 1, where $i_{c r}$ is a critical angle of attack that is characterised by sharp changes in the pressure profile on the blade back, $i_{0}$ is angle of attack that is characterised by correlation of normal and separated performance and is determined by coincidence of the pressure profile of the pressure distribution on the blade back at normal and separated characteristics, $\bar{i}^{\prime}=i^{\prime} / i_{K P}$ is a parameter of inability to calculate the flow performances by the angle of attack (at $i^{\prime}>i^{\prime}{ }_{K P}$ ), and $\bar{i}_{0}=i_{0} / i_{K P}$ is a parameter of inability to calculate (at $\left.i_{0}<i_{K P}\right)$.

Introduction of parameters $\overline{i^{\prime}}$ and $i_{0}^{\prime}$ is stipulated by the fact that as the research results demonstrate the effect of hysteresis in the quasi-stationary problem statement occurs at different inabilities to calculate, which is determined by the specifics of the flow in grids with different geometry.

Figure 5 presents the dependence $\overline{i_{0}}=f\left(\overline{i^{\prime}}\right)$, the analysis of which demonstrates that hysteresis by critical angle of attack occurs in different values of inability to calculate the flow at $\overline{i^{\prime}}>1$.

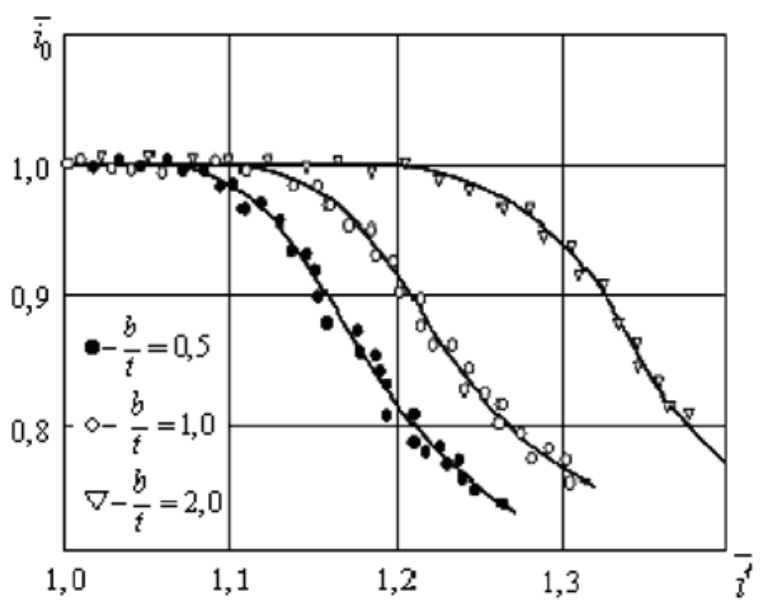

Fig. 5. Dependence of hysteresis on inability to calculate grid streamlining

For the grids with solidity $b / t<0.5$, the effect of hysteresis occurs when angle of attack $i^{\prime}$ exceeds the critical value $i_{K P}$ by $10-20 \%$, which is in this case during transition of the grid from supercritical modes of flow to subcritical (non-separated) angles of attack due to which the initial non-separated flow is recovered. It is $3-18 \%$ less than $i_{K P}$. For the grids with solidity $b / t \leq 1.0$, the effect of hysteresis occurs when the inability to calculate $\overline{i^{\prime}}=1.12-1.25$ and is expressed in the decrease in $i_{0}$ of $4-20 \%$ (within the range of angle of attack change $i_{0}<i<i_{K P}$ ). For the grids with solidity $b / t \leq 2.0$, the effect of hysteresis occurs at inability to calculate $\overline{i^{\prime}}=1.25-1.35$ and is expressed in decrease in $i_{0}$ of $2-13 \%$ (within the range of angle of attack change $\left.i_{0}<i<i_{K P}\right)$.

At values of the parameter of the inability to calculate $\overline{i^{\prime}}<1$ during experiments, the tendency for hysteresis to occur was not detected in corresponding grids.

Let us analyse the occurrence of the effect of hysteresis in typical diffuser grids at inability to calculate the flow parameters $\overline{i^{\prime}}>1.25$ (Fig. 6).

The effect of hysteresis is considered to be negligible when the grid aerofoils have the angle of mean chord curvature equal to $\theta=30^{\circ}$ and the distance between the grids is from $b / t=0.5$ to $b / t=2.0$.

Taking into account that $b / t=0.5$ and $\overline{i^{\prime}}=1.25$, the occurrence of hysteresis can be connected with angle of attack $i_{0}$ at which outgoing streamlining recovery takes place. If $i_{0}$ is $40-48 \%$ lower than $i_{K P}$, then at $b / t=2.0$ this discrepancy yields only $8-12 \%$.

At low level of solidity $(b / t \leq 1.0)$, the increase in the slipping angle from $\varphi=0^{0}$ to $\varphi=30^{0}$ causes $i_{0}$ to decrease correspondingly from 0.6 to 0.52 . When $b / t>1.5$, the increase in the slipping angle causes a less evident occurrence of hysteresis: $i_{0}$ changes from 0.85 to 0.98 . As the research results of the grids consisting of aerofoils with different curvature angles $\left(\theta_{1}=10^{0} ; \theta_{2}=20^{0}\right.$; $\left.\theta_{3}=30^{\circ}\right)$ have shown, the increase in the curvature angle 
of grid aerofoils leads to a more obvious display of the effect of hysteresis at low and high solidity. It is the most intensive influence in grids with low solidity.
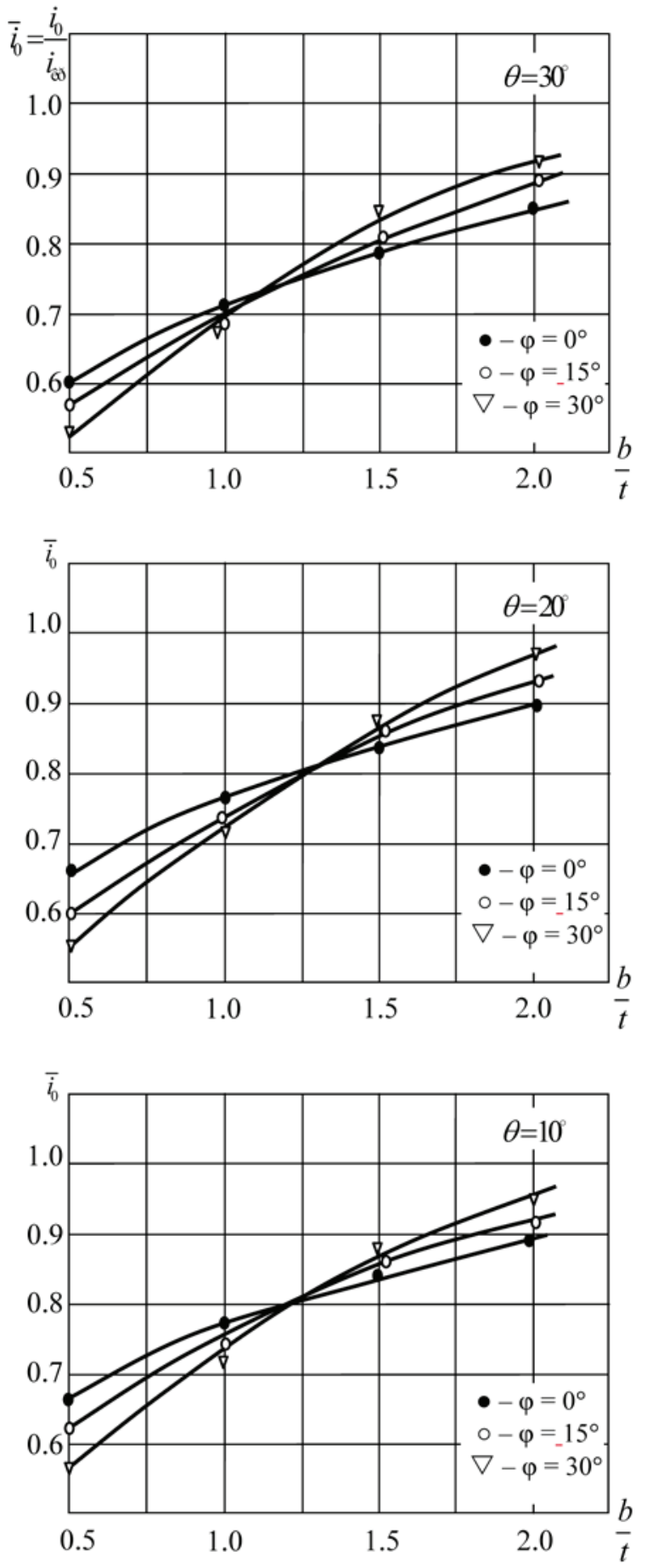

Fig. 6. Hysteresis of diffuser grid performance

\section{Conclusions}

1. General analysis of data regarding experimental research allows one to make a conclusion about the fact that, at the values of the parameter of the inability to calculate $\bar{i}^{\prime}>1.1-1.2$ during the performance of typical compressor grids, hysteresis causes a decrease in the efficiency of compressor grids when they operate in flow that has certain angular non-uniformity at subcritical and critical angles of attack.

2. The improvement in the efficiency of the compressors of gas-turbine engines and the aerodynamic performance of their air-gas channel suggests a solution to the problems regarding the decrease in the influence of the effects of hysteresis on the performances of bladed disks that operate in real conditions under periodic angular non-uniformity of flow.

\section{References}

Chang, P. K. 1979. Control of Flow Separation. Moscow: Mir. 365 p. (in Russian).

Lastivka, I. 2011. Raising gas-dynamic stability margin of axial and centrifugal compressor stages by means of vaned diffuser boundary layer control, Aviation 15(3): 76-81. http://dx.doi.org/10.3846/16487788.2011.624262

Liblein, S.; Randebuseh, W. 1956. Theoretical Loss Relations for Low-Speed Two-Dimensional-Cascade Flow. NASA. 3362.

Kestin, I.; Maeders, P.; Wang, H. 1961. On the boundary layers oscillated with oscillating streams, Applied Scientific Research 10(1): 23-36.

Schlichting, G. 1969. Boundary Layer Theory. Moscow: Nauka. 413 p. (in Russian).

Tereshchenko, Y.; Lastivka, I.; Volianskaya, L., et al. 2010. Flow separation range expansion in gas-turbine engine compressors, East-European Journal of Advanced Technologies 4/7(46): 12-15 (in Russian).

Tereshchenko, Y.; Volianskaya, L.; Kulyk, M., et al. 2005. Theory of Aircraft Gas-Turbine Engines. Kiev: NAU. 500 p. (in Russian). 